\title{
Optical inactivation of the anterior cingulate cortex modulate descending pain pathway in a rat model of trigeminal neuropathic pain created via chronic constriction injury of the infraorbital nerve
}

This article was published in the following Dove Press journal:

Journal of Pain Research

3 October 2017

Number of times this article has been viewed

\author{
Hyeong Cheol Moon' \\ Won Ik Heo ${ }^{2}$ \\ Yon Ji Kim ${ }^{3}$ \\ Daae Lee ${ }^{4}$ \\ So Yoon Won ${ }^{5}$ \\ Hong Rae Kim' \\ Seung Man $\mathrm{Ha}^{\prime}$ \\ Youn Joo Lee ${ }^{6}$ \\ Young Seok Park' \\ 'Department of Medical Neuroscience \\ and Neurosurgery, College of \\ Medicine, ${ }^{2}$ Department of Veterinary, \\ College of Veterinary Medicine, \\ ${ }^{3}$ Department of Biology, College \\ of Natural Sciences, ${ }^{4}$ Department \\ of Advanced Material Engineering, \\ College of Engineering, ${ }^{5}$ Biochemistry \\ and Medical Research Center, \\ Chungbuk National University, \\ Cheongju, 'Department of Radiology, \\ Daejoen St. Mary's Hospital, The \\ Catholic University of Korea, Seoul, \\ Republic of Korea
}

Correspondence: Young Seok Park Department of Medical Neuroscience and Neurosurgery, College of Medicine, Chungbuk National University Hospital, Chungbuk National University, 776, I Sunhwan-ro, Seowon-gu, Cheongju-si 28644, Chungbuk, Republic of Korea Tel +82432696080 Fax +82 $43273 \quad 1614$ Email youngseokparkmd@gmail.com
Purpose: The anterior cingulate cortex (ACC) plays a critical role in the initiation, development, and maintenance of neuropathic pain. Recently, the effects of optical stimulation on pain have been investigated, but the therapeutic effects of optical stimulation on trigeminal neuralgia (TN) have not been clearly shown. Here, we investigated the effects of optical inhibition of the ACC on TN lesions to determine whether the alleviation of pain affects behavior performance and thalamic neuron signaling.

Materials and methods: TN lesions were established in animals by generating a chronic constriction injury of the infraorbital nerve, and the animals received injections of AAV-hSyneNpHR3.0-EYFP or a vehicle (phosphate-buffered saline [PBS]) in the ACC. The optical fiber was fixed into the ipsilateral ACC after the injection of adeno-associated virus plasmids or vehicle. Behavioral testing, consisting of responses to an air puff and cold allodynia, was performed, and thalamic neuronal activity was monitored following optical stimulation in vivo. Optical stimulation experiments were executed in three steps: during pre-light-off, stimulation-light-on, and post-light-off states. The role of the optical modulation of the ACC in response to pain was shown using a combination of optical stimulation and electrophysiological recordings in vivo.

Results: Mechanical thresholds and facial cold allodynia scores were significantly improved in the TN lesion group during optical stimulation compared to those in the control group. Thalamic neuronal activity, consisting of the firing rate (spikes/s) and burst rate (bursts/s), was also decreased during optical stimulation.

Conclusion: Reciprocal optical inhibition of the ACC can alleviate pain-associated behavior and decrease abnormal thalamic sensory neuron activity in the trigeminal neuropathic rat model. The descending pain pathway can modulate thalamic neurons from the ACC following optical stimulation.

Keywords: optogenetics, trigeminal neuralgia, anterior cingulate cortex, neuropathic pain

\section{Introduction}

Trigeminal neuralgia (TN) is a chronic neuropathic pain disorder and is known to be the one of the most painful diseases. It is characterized by relapsing intense sharp pain, numbness, and crawling or unpleasant sensations in the trigeminal area; however, the precise mechanism and fundamental treatment of this neuropathic disorder have not been fully determined. ${ }^{1}$ Characteristics of typical description of TN include mechanical allodynia, which is a hallmark of neuropathic pain. ${ }^{2}$ 
The anterior cingulate cortex (ACC) is a cortical structure involved in processing pain affect with a limited role in sensory processing. ${ }^{3,4}$ The ACC is involved in the modulation and processing of pain affect, and these manipulations of the ACC alter the affective component of pain processing. ${ }^{5}$ It is one of the minimally invasive alternatives to drugs for testing painful conditions. ${ }^{6}$ Support for the contribution of the ACC in pain affect can be found in experiments with rodent subjects. ${ }^{7}$ The ACC is a critical component of the neuroanatomical circuit involved in coordinating the appropriate behavioral response to threatening or noxious stimuli in the environment. ${ }^{8}$ The ACC is thought to contribute to the unpleasantness and salience of pain and the regulation of emotional information. ${ }^{9}$

The light-activated chloride pump halorhodopsin from Natronomonas pharaonis (NpHR) is hyperpolarized and inhibits the propagation of action potentials upon exposure to yellow light; it has been utilized for bidirectional control and is integrated with behavior. ${ }^{10-13}$ The ability to use light to inhibit neurons may have practical applications in basic science, as the NpHR system may be genetically targeted in specific classes of neurons involved in disease processes to enable precise optical therapeutic treatments. ${ }^{11}$ Neuronal activity related to various types of pain has been modulated using optogenetic tools in previous studies. ${ }^{14-16}$

In this study, we monitored pain-related behaviors in a rat model of trigeminal neuropathic pain generated by the chronic constriction of the rat infraorbital nerve. We examined whether the optical stimulation of halorhodopsin $(\mathrm{NpHR})$ in the ACC relieved pain from TN lesions and accessed the modulation of neuronal activity in the thalamus.

\section{Materials and methods}

\section{Animals}

All animal experiments were performed in accordance with the approval of national guidelines and the National Institutes of Health guidelines and were approved by the ethic review committee of Chungbuk National University (CBNUR-107217). Adult female Sprague Dawley rats (200-220 g; Dea
Han Bio Link, Eumseong, South Korea) were maintained under regular housing conditions (12-12 h/light-dark cycle) and allowed access to laboratory food pellets and water ad libitum. Animal testing was performed using a randomized, double-blind, controlled animal trial, and animals were assigned a group designation on the same day. The timeline of experimental protocol is shown in Figure 1. A cage was randomly chosen from the pool of all cages and was not handled by the surgeon. Animals were removed from the cage for use in this study and given a permanent designation by the registrar.

\section{Surgical preparation for TN models}

The rats were randomly allocated to the sham-operated $(n=10)$ or the TN-lesioned $(n=14)$ groups. We generated a rat model of trigeminal neuropathic pain model by ligating the right infraorbital nerve. Rats were anesthetized with a mixture of $15 \mathrm{mg} / \mathrm{kg}$ Zoletil50 ${ }^{\circledR}$ (tiletamine/zolazepam; Virbac Laboratories, Carros, France) and $9 \mathrm{mg} / \mathrm{kg}$ Rompun ${ }^{\circledR}$ (xylazine; Bayer AG, Leverkusen, Germany) in saline and were mounted onto the surgical field. Surgical procedures were performed with the assistance of a surgical microscope (SWF 10X/22; WESCO, Pittsburgh, PA USA) for visual guidance.

The right infraorbital nerve under the skull was exposed in anesthetized rats using sterilized scissors, and a piece of 3-0 nylon was loosely tied twice around the trigeminal nerve using microscope. ${ }^{17-19}$ Sham-operated animals were treated similarly, but the trigeminal nerve was not injured. The exact sample size $(n=10)$ required for contrasts was determined using the PASS 14 software (NCSS Inc., Kaysville, UT, USA; $a=0.05$, power $=0.8$ ). The surgeon successfully completed the operation, and the animals were delivered to the registrar. After surgery, animals were allowed to recover and were observed in a controlled environment.

\section{Injection of adeno-associated viruses and implantation of optical fiber}

The adeno-associated viral (AAV) vector plasmid AAVhSyn-eNpHR3.0-enhanced yellow fluorescent protein

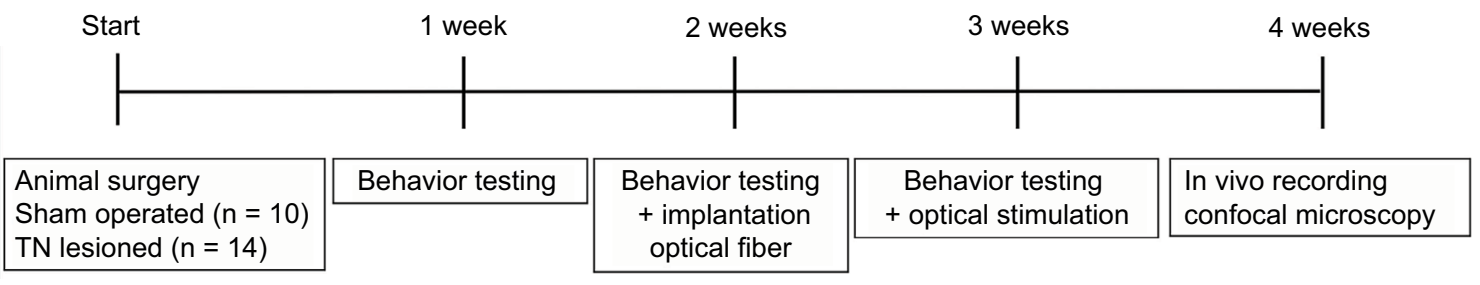

Figure I Timeline of this study.

Abbreviation: $\mathrm{TN}$, trigeminal neuralgia. 
(EYFP) (Korea Institute of Science and Technology, Seoul, South Korea) or phosphate-buffered saline (PBS) was injected into the ACC (AP, bregma, $+2.6 \mathrm{~mm}$; ML, midline, $+0.6 \mathrm{~mm}$; DV, skull, $-2.8 \mathrm{~mm}$ ) to evaluate the behavioral changes and neuronal activity induced by optical stimulation. We randomly divided the animals into two groups: 1) the vehicle control group $(n=4)$ and 2$)$ the NpHR group $(n=10)$. Animals in the control group received a $3-\mu \mathrm{L}$ injection of PBS. Animals in the NpHR group received a 3- $\mu \mathrm{L}$ injection of AAV-hSyn-eNpHR3.0-EYFP targeted to the ipsilateral ACC. The concentration of the viral vectors was $2.3 \times 10^{13}$ genome copies $(\mathrm{GC}) / \mathrm{mL} .{ }^{20}$ We made an incision in the skin over the skull and drilled a hole in the skull to inject the AAV plasmids or PBS into the ACC using a Hamilton syringe and an automated microsyringe pump (Harvard Apparatus, Holliston, MA, USA) at a flow rate of $0.5 \mu \mathrm{L} / \mathrm{min}^{21}$ After the injection was complete, the syringe was maintained in place for 10 minutes to allow the solution to absorb.

After the injection of the AAV plasmid or PBS, an optical fiber (200- $\mu \mathrm{m}$ core, $230-\mu \mathrm{m}$ outer diameter, numerical aperture of 0.48 , hard polymer cladding type; Doric Lenses, Québec City, QC, Canada) was implanted into the skull to send a laser pulse to the ipsilateral ACC. The optical fiber was firmly fixed in place with dental cement (Ortho-Jet Pound Package; Lang Dental, Wheeling, IL, USA).

\section{Optical stimulation}

We used a power supply (ADR-700D; ADR, Shanghai, China) with an output power of $10 \mathrm{~mW}$ and a light of wavelength of $589 \mathrm{~nm}$. The laser properties were modulated by a waveform generator (Keysight 33511B-CFG001; Keysight, Santa Rosa, CA, USA) that allowed us to control the frequency, pulse square, and pulse width. The laser intensity was set at $1 \mathrm{~mW}$, with continuous light exposure for optical stimulation. Optical modulation was performed prior to (pre), during (laser-on), and after (post) optical stimulation for 10 minutes during each step.

\section{Behavior tests}

Measurement of mechanical thresholds in the air-puff tests

Rats were place in a plexiglas cage $(20 \times 20 \times 14 \mathrm{~cm})$ with a grid bottom and were habituated to the environment for at least 30 minutes in a quiet place. An air puff (output pressure: $\sim 100 \mathrm{psi}$ ) was administered (duration of continuous air puff, $4 \mathrm{~s}$; duration of interval between puffs, $10 \mathrm{~s}$ ) to the right facial area. The air flowed through a narrow tubing tip and was released at a $90^{\circ}$ angle; the puff emerged $\sim 1 \mathrm{~cm}$ from the face. The presence of aggressive behaviors, such as biting or turning the head, was evaluated. Mechanical thresholds were evaluated and represented $>50 \%$ of the overall responses. The test was stopped if the animal did not respond to a stimulus of at least 40 psi. $^{22}$

\section{Measurement of the mean facial cold allodynia scores}

Rats were placed in the same clear plexiglas cage describe earlier and were allowed to habituate to the environment for 30 minutes in a quiet place. Facial cold allodynia was estimated using the acetone drop method. ${ }^{23}$ We dropped acetone $(100 \mathrm{~mL})$ on the ipsilateral side of the face using a glass syringe while counting the number of indicators, such as scratching or rubbing, that occurred during $2 \mathrm{~min}$. This assessment did not include parts of the body other than the face. The pain response behavior was assessed on each day before and after surgery. ${ }^{24}$

\section{Extracellular recordings in vivo}

At least 2 weeks after optical fiber implantation, animals in the vehicle group $(n=4)$ and the NpHR group $(n=4)$ that had received TN lesions were anesthetized with $15 \mathrm{mg}$ / $\mathrm{kg}$ tiletamine/zolazepam and $9 \mathrm{mg} / \mathrm{kg}$ xylazine in saline. Extracellular recordings were obtained from the ventral posteromedial nucleus (AP: $-3.5 \mathrm{~mm}$; ML: $+2.8 \mathrm{~mm}$; DV: $-6.0 \mathrm{~mm}$ ) using a quartz-insulated carbon electrode (Kation Scientific, Minneapolis, MN, USA). We recorded neuronal activity, which was digitized $(40 \mathrm{kHz})$ with a Digital Lynx SX data acquisition system (Neuralynx Inc., Bozeman, MT, USA). Similar standard neuronal waveform shapes in the recorded neuronal activity were identified, and selected similar patterns of spike clusters were detected using SpikeSort 3D software (Neuralynx Inc.).

\section{Analysis of the bursting and firing rates}

The activity in thalamic neurons can be divided into bursts rates (bursts/s) and overall firing rates (spikes/s), according to the type of optical stimulation, using NeuroExplorer software (Neuralynx Inc.). Activity was assessed for 10 minutes in each state: pre, laser-on, and post states. We defined burst rates as a group of spikes with a maximum $4 \mathrm{~ms}$ interval between spikes and a minimum of three spikes, with a $100 \mathrm{~ms}$ interval between bursts. We compared and selected similar interspike interval histograms. 


\section{Confocal microscopy}

Rats were transcardially perfused with $0.9 \%$ saline containing 10,000 IU of heparin (Hanlim Pharm, Seoul, South Korea), followed by $4 \%$ paraformaldehyde (Biosesang, Seongnam, South Korea). Brains were extracted and fixed overnight in $4 \%$ paraformaldehyde, followed by dehydration in $30 \%$ sucrose. Coronal sections of the ACC were cut on a cryotome (Thermo Fisher Scientific, Waltham, MA, USA). Free-floating brain slices were preserved in $0.08 \%$ sodium azide (Sigma-Aldrich Co., St Louis, MO, USA) in PBS at $4^{\circ} \mathrm{C}$. A confocal microscope (Carl Zeiss Meditec AG, Jena, Germany) with ZEN microscope software (LSM710 system; Carl Zeiss Meditec AG) was used to capture the images of fluorescent.

\section{Statistical analysis}

Data were analyzed using GraphPad Prism (GraphPad Software, Inc., San Diego, CA, USA), and data from the behavior test and the in vivo recordings are shown as mean \pm standard deviation (SD). We performed either an unpaired $t$-test, two-way analysis of variance (ANOVA) with Tukey's post hoc test, or a repeated measures test, depending on the conditions of the experiment. Behavioral tests were assessed based on the mean values for each of the three optical states. Unpaired $t$-tests were used to compare firing rate between $\mathrm{TN}$ and sham-operated animals.

\section{Results}

\section{Behavioral responses to mechanical thresholds and facial cold allodynia after chronic constriction injury of the rat infraorbital nerve}

We measured the air-puff thresholds (psi) and counted the indicator scores after the cold acetone test to assess the induction of mechanical thresholds and facial cold allodynia in the TN-lesioned animals. The animals with $\mathrm{TN}$ showed a decrease in their thresholds and increased indicator scores compared with sham-operated animals. TN lesions significantly decreased mechanical thresholds from $22.00 \pm 3.65$ psi to $8.80 \pm 3.79 \mathrm{psi}$, with a two-way ANOVA showing a significant difference after surgery, $F(1,36)=91.33, p<0.001$, and the cold allodynia scores increased significantly from $13.10 \pm$ 3.63 to $23.50 \pm 1.78$ (mean $\pm \mathrm{SD}$ ), with a two-way ANOVA also showing a significant difference after surgery, $F(1,36)=$ 105.2, $p<0.001$. An ANOVA with Tukey's post hoc test was used to determine whether there were differences between different groups and days (Figure 2A and B).

\section{Behavioral responses associated with mechanical thresholds and cold allodynia in response to optical stimulation of the ACC}

We assessed the facial allodynia-associated behaviors after 3 weeks following the injection of either AAV-hSyneNpHR3.0-EYFP or vehicle and optical stimulation of the ACC. We used the air-puff test and cold allodynia scores to examine facial allodynia. Optical stimulation of the ACC increased mechanical thresholds in the TN-NpHR animals $(24.00 \pm 3.55$ at the pre state, $7.20 \pm 5.35$ at the yellow laser-on state, and $13.00 \pm 4.19$ at the post state [mean $\pm \mathrm{SD}]$ ) compared to the sham-operated animals $(23.50 \pm 1.78$ at the pre state, $23.40 \pm 1.95$ at the yellow laser-on state, and $24.00 \pm 3.55$ at the post state). A two-way ANOVA showed significant effects of optical stimulation, $F(2,81)=180.9, p<0.001$, and differences between groups, $F(2,81)=12.24, p<0.001$ (Figure 3A). Facial allodynia scores decreased in the TN-NpHR animals (4.20 $\pm 0.63 \mathrm{psi}$ at the pre state, $15.6 \pm 6.38$ at the yellow laser-on state, and $6.80 \pm 2.53$ at the post state $[p<0.05])$ compared to the scores for the sham-operated animals $(5.40 \pm 2.11 \mathrm{psi}$ at the pre state, $6.00 \pm 2.10$ at the yellow laser-on state, and $7.60 \pm 2.21$ at the post state; Figure 3B). A two-way ANOVA showed significant effects of optical stimulation, $F(2,81)=$ $58.31, p<0.001$, and differences between the groups, $F(2,81)$ $=17.9, p<0.001$. Optical stimulation of the ACC with a yellow laser increased the air-puff thresholds in TN animals expressing NpHR but not in the vehicle-treated animals.

\section{Neuronal activity in the ventral posteromedial thalamus following optical stimulation of the ACC using $\mathrm{NpHR}$}

To confirm the neuronal activity, we monitored firing rates of posteromedial thalamic neurons between sham-operated $(\mathrm{n}=4)$ and TN-lesion $(\mathrm{n}=8)$ rats. Spontaneous firing rates were significantly higher in TN-lesion rats (14.28 \pm 7.7 spikes/s) compared with those in sham-operated rats (7.81 \pm 4.43 spikes/s, unpaired $t$-test, $p<0.01$; Figure 4A).

We assessed the effects of optical stimulation of the ACC in the TN-NpHR group $(\mathrm{n}=4)$, and the thalamic firing rates were $13.43 \pm 6.36$ at the pre state, $4.80 \pm 4.46$ at the yellow laser-on state, and $12.00 \pm 6.51$ at the post state (Figure 4B). A two-way ANOVA showed significant effects of optical stimulation, $F(2,2142)=641.2, p<0.001$, and differences between the groups, $F(5,2142)=153.9, p<0.001$. Burst rates also appeared to decrease during optical stimulation in the TN-NpHR group; the thalamic burst rates were $0.57 \pm 0.68$ 

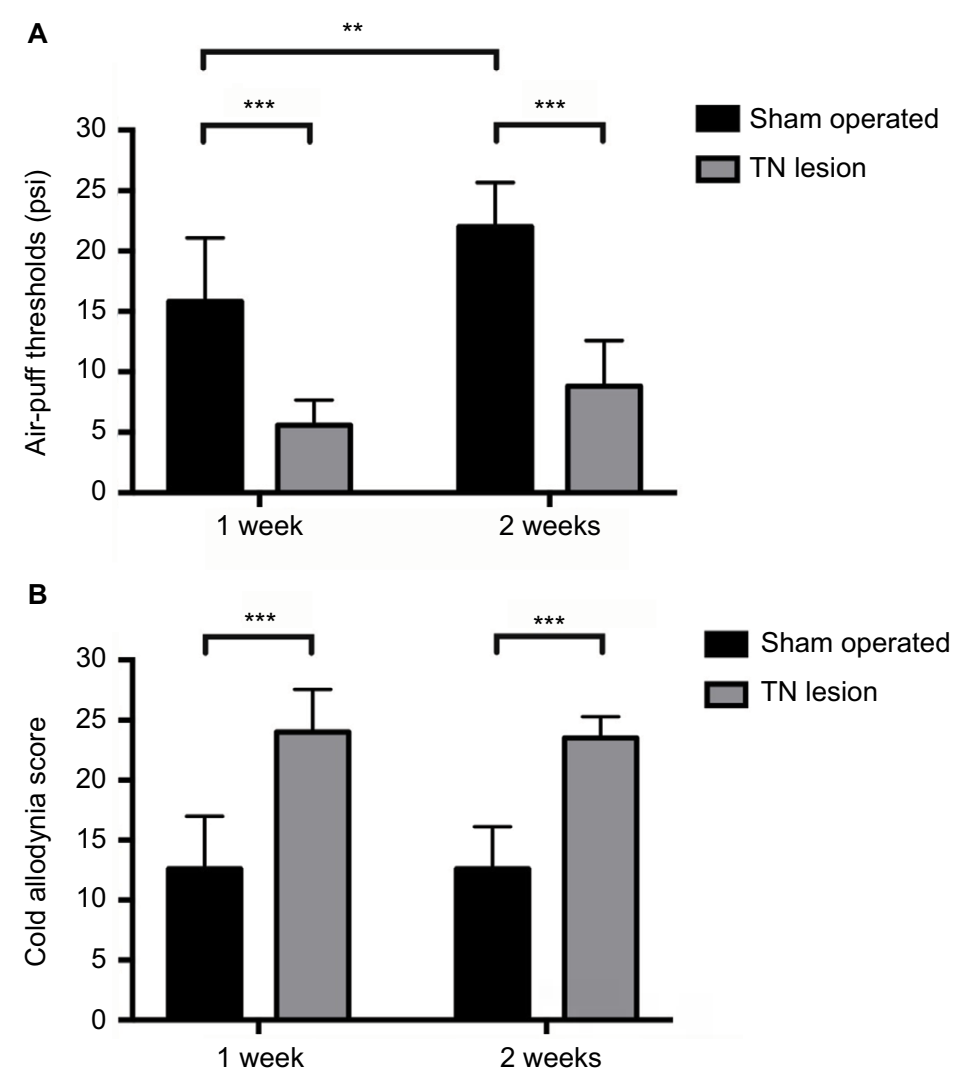

Figure 2 Facial allodynia behavior test.

Notes: Changes in the mechanical thresholds of the sham-operated and TN-lesioned rats were assessed for 2 weeks using air puffs. TN-lesioned rats $(\mathrm{n}=14)$ responded at significantly lower ipsilateral mechanical thresholds than sham-operated rats $(n=10)(\mathbf{A})$. Cold allodynia scores for sham-operated and TN-lesioned rats were obtained over 2 weeks by dropping acetone on the animal's face. TN-lesioned rats exhibited a significant increase in ipsilateral facial allodynia compared to sham-operated rats (B). All data represent the mean \pm SD values. The two-way ANOVA followed by Tukey's post hoc test was used. $* * p<0.01$, and $* * * p<0.00$ I for comparisons between each groups. Abbreviations: TN, trigeminal neuralgia; SD, standard deviation; ANOVA, analysis of variance.

at the pre state, $0.38 \pm 0.71$ at the yellow laser-on state, and $0.44 \pm 0.79$ at the post state (Figure $4 \mathrm{C}$ ). A two-way ANOVA showed significant effects of optical stimulation, $F(2,2394)=$ $5.32, p<0.01$. Optical stimulation of the ACC with a yellow laser decreased thalamic discharge in the NpHR group but not in the sham-operated group. Abnormal thalamic firing reappeared at the post state $(>20 \mathrm{~min})$.

\section{Confocal microscopy}

We observed the expected expression of AAV-hSyneNpHR3.0-EYFP in the ACC and confirmed the correct localization and expression of the optogenetic viral vectors. Confocal imagining revealed that EYFP was highly expressed and localized in the ACC, and we observed that NpHR was highly expressed and localized in the ACC (Figure 5).

\section{Discussion}

Efficient optical stimulation of the ACC using halorhodopsin improved mechanical and cold allodynia and changed the thalamic output in a trigeminal neuropathic pain model.
Importantly, optical stimulation of the ACC has an effect on the thalamus that ultimately affects the pain sensory system.

The ACC is also involved in pain modulation. Emotional states affect the degree of unpleasantness associated with pain, and the magnitude of this effect often correlates with altered pain-evoked ACC activations. ${ }^{25,26}$

The cingulate cortex is a key component of the limbic system, and the anterior cingulate gyrus plays an important role in the integration of nociceptive, motor, affective, and memory functions. ${ }^{27}$ The ACC is of particular importance for the perception and evaluation of the unpleasantness of pain, which is an integral part of the limbic system and is central for processing the emotional aspects of pain. ${ }^{27,28}$ Based on accumulating evidence from both human beings and animals, ACC is important for pain-related perception and thus is likely a target for pain relief therapy. ${ }^{21}$ Synaptic activation in the ACC induced by nerve injury is critical for the generation and maintenance of neuropathic pain, including pain-related synaptic potentiation, and can prevent or alleviate neuropathic pain hypersensitivity. ${ }^{29,30}$ 
A

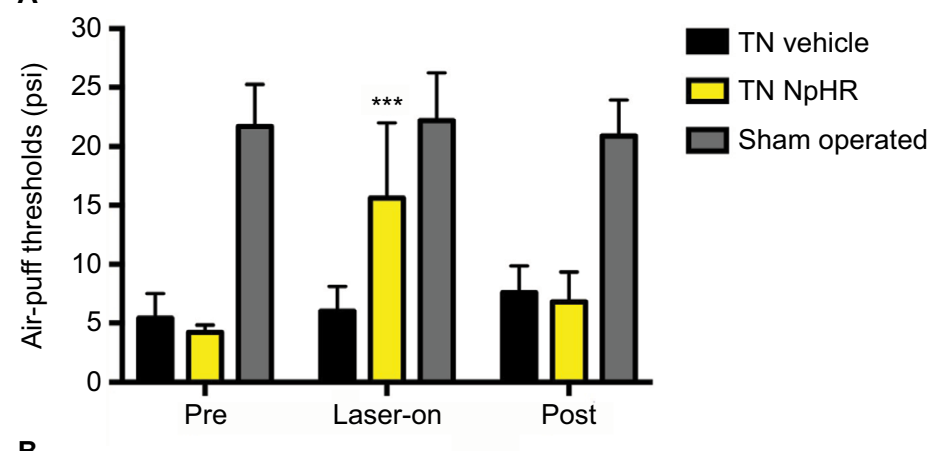

B

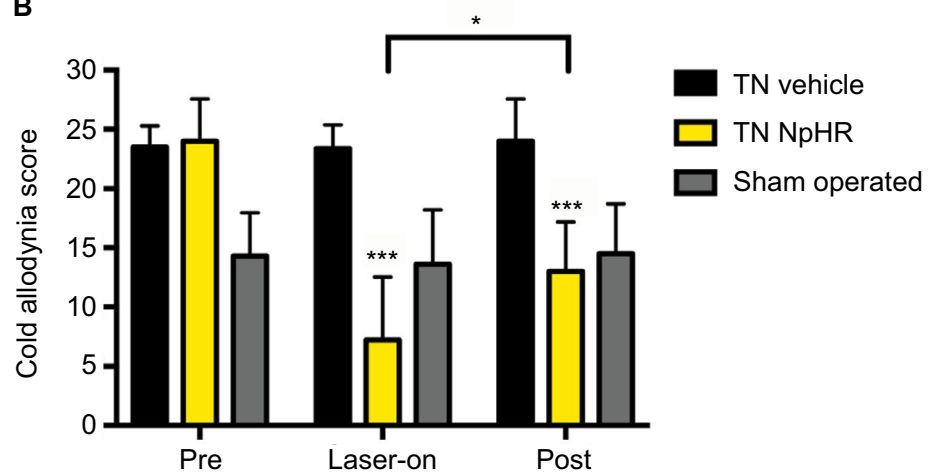

Figure 3 Results of the pain allodynia behavior test according to optical stimulation in the pre, laser-on, and post states.

Notes: Mechanical thresholds were increased $(\mathbf{A})$, and the cold allodynia scores were decreased $(\mathbf{B})$ during optical stimulation. All data are presented as mean \pm SD. The two-way ANOVA followed by Tukey's post hoc test was used. ** $p<0.01$, and *** $p<0.001$ for comparisons between each groups.

Abbreviations: SD, standard deviation; ANOVA, analysis of variance; TN, trigeminal neuralgia; NpHR, Natronomonas pharaonis.

A

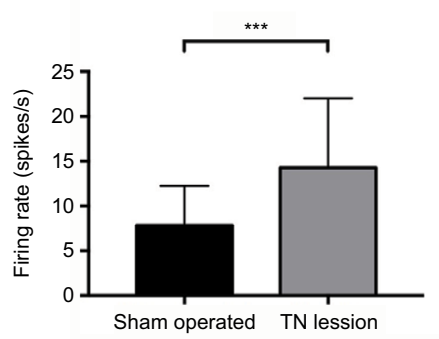

D

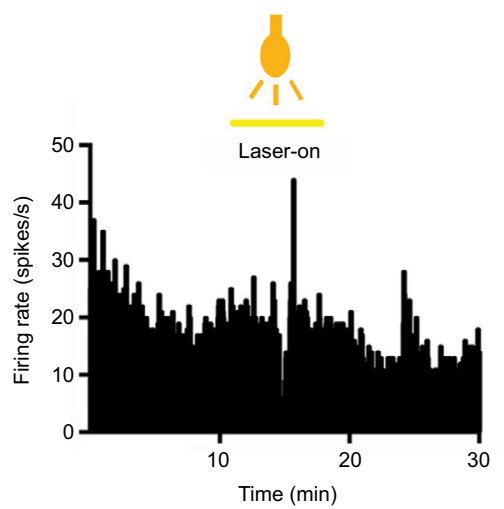

B

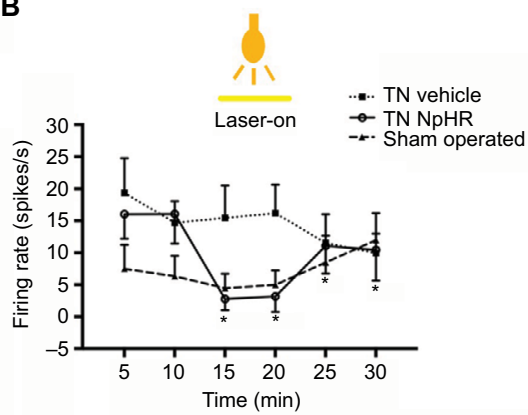

E

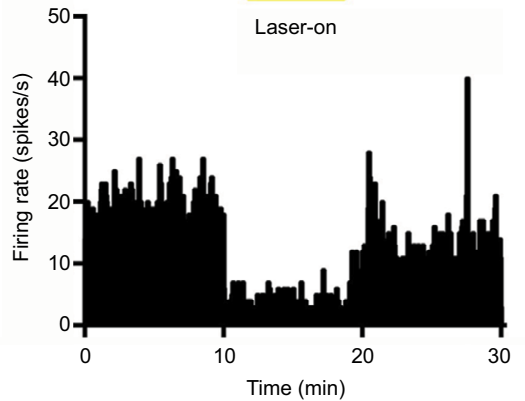

C

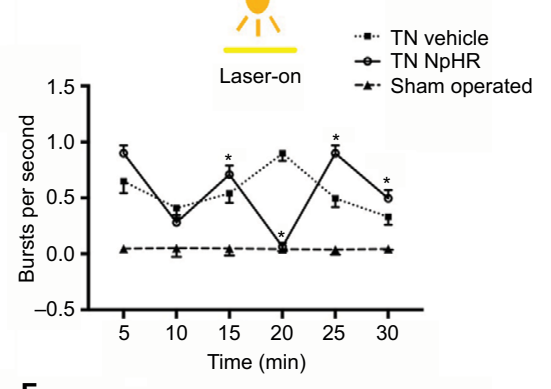

$\mathbf{F}$

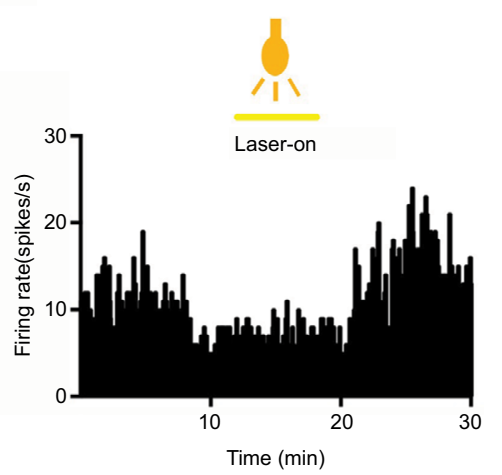

Figure 4 Alterations in thalamic output depending on the optogenetic inhibition of the ACC.

Notes: Differences in spontaneous and evoked firing rates in the neurons of the VPM between sham-operated and TN lesions $(\mathbf{A})(\mathrm{n}=8)$. Overall single neuronal activity in the thalamus by optical stimulation (B). Burst firing rates following optical stimulation (C). Real-time firing rates (histogram) in TN-vehicle (D), TN-NpHR (E), and shamoperated (F) rats. Unpaired $t$-test comparison between sham-operated and TN groups. The two-way ANOVA test was used to compare neuronal activity according to the optical stimulation and different groups.

Abbreviations: ACC, anterior cingulate cortex; TN, trigeminal neuralgia; NpHR, Natronomonas pharaonis; ANOVA, analysis of variance; VPM, ventral posteromedial thalamic nucleus. 

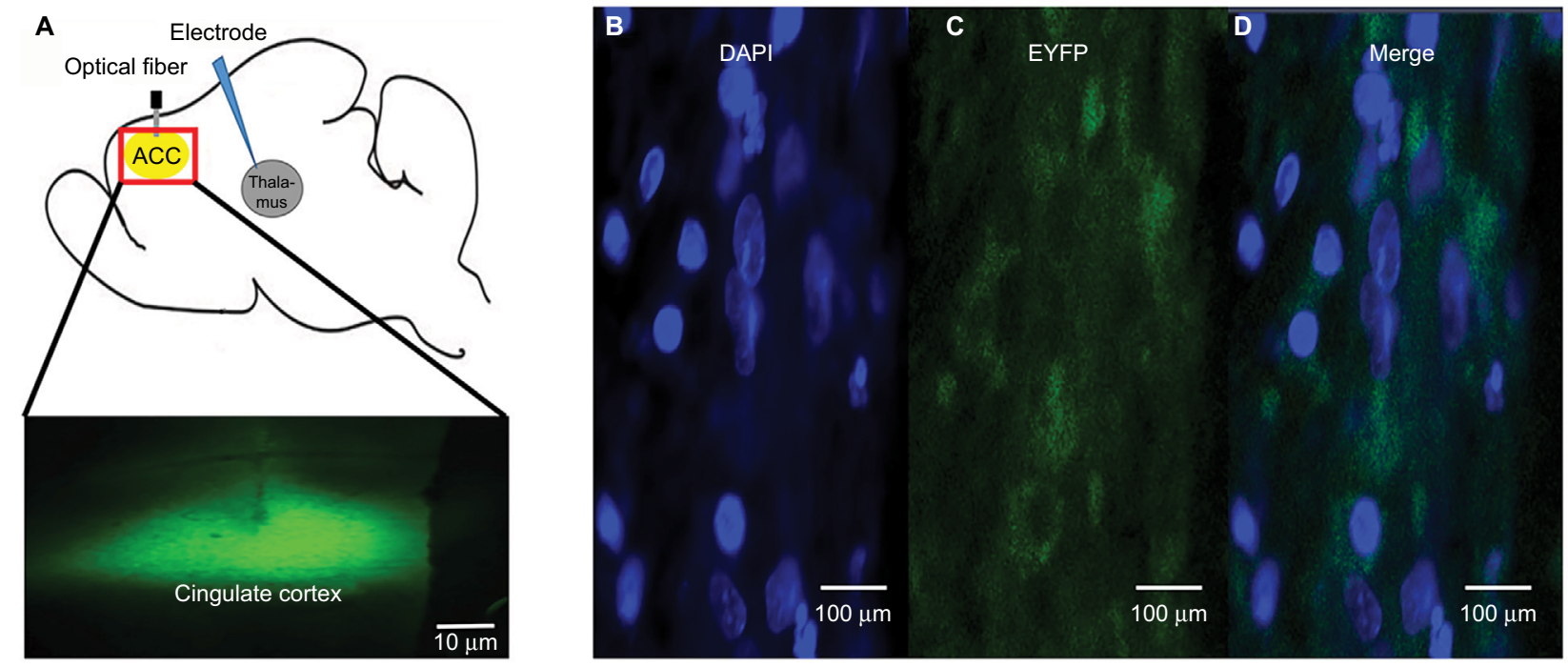

Figure 5 A confocal image shows AAV-hSyn-eNpHR3.0-EYFP expression in the ACC.

Notes: Illustration showing the position of the ACC $(\mathbf{A})$. Fluorescent images show DAPI and EYFP staining, as well as the merge between these markers $(\times 200$; B-D) in the ACC.

Abbreviations: AAV, adeno-associated virus; ACC, anterior cingulate cortex; DAPI, 4',6-diamidino-2-phenylindole; EYFP, enhanced yellow fluorescent protein.

The chronic constriction injury of TN is caused by a proximal compression of the trigeminal nerve root close to the brain stem. TN is comparable to what has previously been described for chronic pain conditions and appears to reflect the cortical adaptation to long-term, high-frequency pain perception..$^{31}$ Activity in the ACC, hippocampus, and temporal lobe is negatively correlated to the disease duration, and these structures may be important for the development of pain. ${ }^{32}$

The TN neuropathic pain model exhibits abnormal thalamic discharge, which increases painful responses to noxious stimuli, similar to that found in peripheral injury models. ${ }^{33}$ This abnormal discharge is modulated by the nociceptive or emotional area in the ACC. As shown in extensive electrophysiological recordings, neurons within the ACC respond to noxious stimuli and are activated during pain anticipation. ${ }^{34,35}$ Electrical stimulation of the medial thalamic nuclei produced an increase in the signal in the ACC, suggesting that the medial thalamus is involved in the affective motivational component of pain. ${ }^{36}$ Electrophysiological data have been presented that describe excitatory responses of neurons to nociceptive stimulation in the ventromedial thalamus. ${ }^{37}$ The thalamic nuclei are included in the sensory discriminative and affective motivational components of pain and receive the different sets of projections from ACC and frontal cortical regions. ${ }^{33,38}$ Thalamic nuclei are suggested to modulate responses to neuropathic pain. It will be particularly interesting to optogenetically control the excitatory to inhibitory ratio in the ACC by targeting glutamatergic (excitatory) and gamma-aminobutyric acid (GABA)ergic (inhibitory) neurons $\mathrm{s}^{39}$ and altering neurotransmitter release probability in ACC synapses in models of chronic pain. ${ }^{40}$ There was some possibility of inhibition of glutamatergic neurons in our study, but the majority of the ACC neurons seem to be inhibitory. ${ }^{21,28}$ Although the ACC-to-thalamic connection in the efferent pathway is still debated, the ACC influences the thalamus indirectly via inhibitory neurons. Neurons in the deep layers of the ACC also send their projections directly or indirectly to the dorsal horn of the spinal cord. ${ }^{41}$ Optical inactivation of GABAergic neuron in ACC influences the descending pain pathway, alleviating trigeminal-associated pain.

Optical stimulation of NpHR-transfected glutamatergic neurons in the ACC is postulated to decrease abnormal thalamic discharge, rather than influencing thalamic disinhibition.

We postulated that NpHR transfection of glutamatergic neurons and the optical stimulation of the ACC via NpHR would result in a decrease in abnormal thalamic discharges rather than thalamic disinhibition.

Cingulotomy has been successfully used to treat intractable cancer ${ }^{42,43}$ and noncancerous pain. ${ }^{44}$ ACC deep-brain stimulation in chronic neuropathic pain in human has been reported to result in pain relief. ${ }^{45}$ Optical inhibition via NpHR acts as an optical scalpel to disrupt the transmission of the abnormal pain signals to the thalamus; we observed a reduction in pain-associated responses and a decrease in spontaneous activity in thalamic neurons.

In the rat model of $\mathrm{TN}$ neuropathic pain rat model, the optogenetic inhibition of the ACC via NpHR altered 
the thalamic discharges and improved pain behaviors. Optogenetic silencing of the principal neurons in organotypic hippocampal cultures has been shown to suppress stimulation-induced burst activity, with silencing achieved by yellow light illumination of slices expressed NpHR. ${ }^{46,47}$ The NpHR system enables the precise optical control of neural circuits, and these fast, genetically based, neuralspike-controlling technologies can powerfully augment existing tools used for evaluating neural systems. ${ }^{48-54}$ Upon light stimulation, NpHR inhibits the generation of action potentials. ${ }^{11}$ Halorhodopsin is a light-gated ion pump, specific for chloride ions. Among its many isoforms, halorhodopsin from NpHR has been used to achieve inhibition of action potentials in neurons in mammalian systems. ${ }^{10}$ Recent optogenetic applications for stimulating indirect pathways in the cortex and thalamus are leading to both revisions and consolidations of previous concepts concerning the direct and indirect pathways. In this study, we optogenetically modulated inhibitory pathways between the ACC and thalamus; therefore, our model is limited to optogenetic examinations of the thalamic inhibitory synapses.

This study has some limitations. We used an AAV-hSyneNpHR3.0-EYFP viral vector, which can nonspecifically transfect both excitatory and inhibitory neurons. Optical stimulation via NpHR may affect both excitatory and inhibitory neurons, and we did not perform immunohistochemistry for specific glutamate or GABA cell types. We cannot state that our results are due to excitatory or inhibitory neuronal function. This needs further study using specific viral promotors or transgenic models. Brain functional and anatomical studies to link the descending pain modulatory system from the brain stem in processing nociceptive pain. ${ }^{55}$ We induced a chronic constriction injury of the infraorbital nerve, performed optical stimulation of the ACC, and electrophysiologically monitored ventral posteromedial nucleus activity in the brain, but we did not monitor activity in the medulla or at the spinal cord level.

\section{Conclusion}

Optical stimulation of the ACC improved mechanical pain and facial cold allodynia and altered the abnormal activity of thalamic neurons in a rat model of trigeminal neuropathic pain. Furthermore, optogenetic inhibition of the ACC might modulate thalamic discharge. Optical inhibition of the ACC via NpHR may serve as an optical scalpel in the trigeminal neuropathic pain model. This conclusion is supported by previous studies of pain behaviors and the results of in vivo electrophysiological studies using NpHR. The role of optical modulation of the ACC in pain in vivo was examined using a combination of optical stimulation and electrophysiological recordings. Optical inactivation of GABAergic neurons in the ACC influence the descending pain pathway, and it decreases abnormal thalamic firing, therefore, alleviating trigeminal pain. The descending pain pathway can modulate thalamic neurons from the ACC in response to optical stimulation.

\section{Acknowledgment}

This work was supported by the National Research Foundation of Korea (NRF-2016H1D5A1908909, NRFR1C1A2A01053318, and NRF-2014K1A3A1A21001372).

\section{Author contributions}

Conceived and designed the experiments: HCM, WIH, and YJK. Performed the experiments: HCM, WIH, YJK, SYW, YJL, and YSP. Analyzed the data: HCM and WIH. Contributed reagents/materials/analysis tools: HCM, WIH, HRK,SMH,YJK, YJL, and YSP. Wrote the paper: HCM, WIH, and YSP. All authors contributed toward data analysis, drafting and revising the paper and agree to be accountable for all aspects of the work.

\section{Disclosure}

The authors report no conflicts of interest in this work.

\section{References}

1. Okeson JP. Bell's Orofacial Pains: The Clinical Management of Orofacial Pain. Chicago, IL, USA: Quintessence Publishing Company; 2005.

2. Liao C, Zhang W, Yang M, Zhong W, Liu P, Li S. Microvascular decompression for trigeminal neuralgia: the role of mechanical allodynia. World Neurosurg. 2016;91:468-472.

3. Vogt BA, Miller MW. Cortical connections between rat cingulate cortex and visual, motor, and postsubicular cortices. J Comp Neurol. 1983;216(2):192-210.

4. Vogt BA. Cingulate cortex. In: Peters A, Johns EG, editors. New York: Plenum Publishing Corporation; 1985.

5. Fuchs PN, Peng YB, Boyette-Davis JA, Uhelski ML. The anterior cingulate cortex and pain processing. Front Integr Neurosci. 2014;8:35.

6. Park SI, Oh JH, Hwang YS, Kim SJ, Chang JW. Electrical stimulation of the anterior cingulate cortex in a rat neuropathic pain model. Acta Neurochir Suppl. 2006;99:65-71.

7. Foltz EL, White LE Jr. Pain "relief" by frontal cingulumotomy. J Neurosurg. 1962;19:89-100.

8. LaBuda CJ, Fuchs PN. Attenuation of negative pain affect produced by unilateral spinal nerve injury in the rat following anterior cingulate cortex activation. Neuroscience. 2005;136(1):311-322.

9. Davis KD, Moayedi M. Central mechanisms of pain revealed through functional and structural MRI. J Neuroimmune Pharmacol. 2013;8(3):518-534.

10. Gradinaru V, Thompson KR, Deisseroth K. eNpHR: a Natronomonas halorhodopsin enhanced for optogenetic applications. Brain Cell Biol. 2008;36(1-4):129-139.

11. Zhang F, Wang LP, Brauner M, et al. Multimodal fast optical interrogation of neural circuitry. Nature. 2007;446(7136):633-639. 
12. Gradinaru V, Thompson KR, Zhang F, et al. Targeting and readout strategies for fast optical neural control in vitro and in vivo. J Neurosci. 2007;27(52):14231-14238.

13. Han X, Boyden ES. Multiple-color optical activation, silencing, and desynchronization of neural activity, with single-spike temporal resolution. PLoS One. 2007;2(3):e299.

14. Bonin RP, Wang F, Desrochers-Couture M, et al. Epidural optogenetics for controlled analgesia. Mol Pain. 2016;12:1744806916629051.

15. Copits BA, Pullen MY, Gereau RW 4th. Spotlight on pain: optogenetic approaches for interrogating somatosensory circuits. Pain. 2016;157(11):2424-2433.

16. Liu S, Li C, Xing Y, Wang Y, Tao F. Role of neuromodulation and optogenetic manipulation in pain treatment. Curr Neuropharmacol. 2016;14(6):654-661

17. Piovesan EJ, Oshinsky M, Silberstein S, Kowacs PA, Novak EM, Werneck LC. Botulinum neurotoxin type-A when utilized in animals with trigeminal sensitization induced a antinociceptive effect. Arq Neuropsiquiatr. 2016;74(6):462-469.

18. Chichorro JG, Zampronio AR, Souza GE, Rae GA. Orofacial cold hyperalgesia due to infraorbital nerve constriction injury in rats: reversal by endothelin receptor antagonists but not non-steroidal anti-inflammatory drugs. Pain. 2006;123(1-2):64-74.

19. Vos BP, Strassman AM, Maciewicz RJ. Behavioral evidence of trigeminal neuropathic pain following chronic constriction injury to the rat's infraorbital nerve. J Neurosci. 1994;14(5 pt 1):2708-2723.

20. Zhang Z, Feng J, Wu C, Lu Q, Pan ZH. Targeted expression of channelrhodopsin-2 to the axon initial segment alters the temporal firing properties of retinal ganglion cells. PLoS One. 2015;10(11):e 0142052

21. Gu L, Uhelski ML, Anand S, et al. Pain inhibition by optogenetic activation of specific anterior cingulate cortical neurons. PLoS One. 2015;10(2):e0117746.

22. Ollerenshaw DR, Bari BA, Millard DC, Orr LE, Wang Q, Stanley GB Detection of tactile inputs in the rat vibrissa pathway. J Neurophysiol. 2012;108(2):479-490.

23. Sideris A, Piskoun B, Russo L, Norcini M, Blanck T, Recio-Pinto E. Cannabinoid 1 receptor knockout mice display cold allodynia, but enhanced recovery from spared-nerve injury-induced mechanical hypersensitivity. Mol Pain. 2016;12:1744806916649191.

24. Yamamoto S, Ono H, Kume K, Ohsawa M. Oxaliplatin treatment changes the function of sensory nerves in rats. $J$ Pharmacol Sci. 2016;130(4):189-193.

25. Bushnell MC, Ceko M, Low LA. Cognitive and emotional control of pain and its disruption in chronic pain. Nat Rev Neurosci. 2013;14(7):502-511.

26. Villemure C, Bushnell MC. Mood influences supraspinal pain processing separately from attention. J Neurosci. 2009;29(3):705-715.

27. Vogt BA. Pain and emotion interactions in subregions of the cingulate gyrus. Nat Rev Neurosci. 2005;6(7):533-544.

28. Blom SM, Pfister JP, Santello M, Senn W, Nevian T. Nerve injuryinduced neuropathic pain causes disinhibition of the anterior cingulate cortex. J Neurosci. 2014;34(17):5754-5764.

29. Shen FY, Chen ZY, Zhong W, et al. Alleviation of neuropathic pain by regulating T-type calcium channels in rat anterior cingulate cortex. $\mathrm{Mol}$ Pain. 2015;11:7.

30. Li XY, Ko HG, Chen T, et al. Alleviating neuropathic pain hypersensitivity by inhibiting PKMzeta in the anterior cingulate cortex. Science. 2010;330(6009):1400-1404.

31. Rodriguez-Raecke R, Niemeier A, Ihle K, Ruether W, May A. Brain gray matter decrease in chronic pain is the consequence and not the cause of pain. J Neurosci. 2009;29(44):13746-13750.

32. Obermann M, Rodriguez-Raecke R, Naegel S, et al. Gray matter volume reduction reflects chronic pain in trigeminal neuralgia. Neuroimage. 2013;74:352-358.

33. Ab Aziz CB, Ahmad AH. The role of the thalamus in modulating pain. Malays J Med Sci. 2006;13(2):11-18.
34. Kuo CC, Yen CT. Comparison of anterior cingulate and primary somatosensory neuronal responses to noxious laser-heat stimuli in conscious, behaving rats. $J$ Neurophysiol. 2005;94(3):1825-1836.

35. Koyama T, Kato K, Mikami A. During pain-avoidance neurons activated in the macaque anterior cingulate and caudate. Neurosci Lett. 2000;283(1):17-20.

36. Shyu BC, Lin CY, Sun JJ, Chen SL, Chang C. BOLD response to direct thalamic stimulation reveals a functional connection between the medial thalamus and the anterior cingulate cortex in the rat. Magn Reson Med. 2004;52(1):47-55.

37. Monconduit L, Bourgeais L, Bernard JF, Villanueva L. Convergence of cutaneous, muscular and visceral noxious inputs onto ventromedial thalamic neurons in the rat. Pain. 2003;103(1-2):83-91.

38. Shibata H, Naito J. Organization of anterior cingulate and frontal cortical projections to the anterior and laterodorsal thalamic nuclei in the rat Brain Res. 2005;1059(1):93-103.

39. Yizhar O, Fenno LE, Prigge M, et al. Neocortical excitation/inhibition balance in information processing and social dysfunction. Nature. 2011;477(7363):171-178.

40. Zhao MG, Ko SW, Wu LJ, et al. Enhanced presynaptic neurotransmitter release in the anterior cingulate cortex of mice with chronic pain. J Neurosci. 2006;26(35):8923-8930.

41. Bliss TV, Collingridge GL, Kaang BK, Zhuo M. Synaptic plasticity in the anterior cingulate cortex in acute and chronic pain. Nat Rev Neurosci. 2016;17(8):485-496.

42. Hassenbusch SJ, Pillay PK, Barnett GH. Radiofrequency cingulotomy for intractable cancer pain using stereotaxis guided by magnetic resonance imaging. Neurosurgery. 1990;27(2):220-223.

43. Viswanathan A, Harsh V, Pereira EA, Aziz TZ. Cingulotomy for medically refractory cancer pain. Neurosurg Focus. 2013;35(3):E1.

44. Wilkinson HA, Davidson KM, Davidson RI. Bilateral anterior cingulotomy for chronic noncancer pain. Neurosurgery. 1999;45(5):1129-1134; discussion 1134-1126.

45. Boccard SG, Pereira EA, Moir L, et al. Deep brain stimulation of the anterior cingulate cortex: targeting the affective component of chronic pain. Neuroreport. 2014;25(2):83-88.

46. Berglind F, Ledri M, Sorensen AT, et al. Optogenetic inhibition of chemically induced hypersynchronized bursting in mice. Neurobiol Dis. 2014;65:133-141.

47. Tonnesen J, Sorensen AT, Deisseroth K, Lundberg C, Kokaia M. Optogenetic control of epileptiform activity. Proc Natl Acad Sci USA. 2009;106(29):12162-12167.

48. Faumont S, Lockery SR. The awake behaving worm: simultaneous imaging of neuronal activity and behavior in intact animals at millimeter scale. J Neurophysiol. 2006;95(3):1976-1981.

49. Rosenmund C, Sigler A, Augustin I, Reim K, Brose N, Rhee JS. Differential control of vesicle priming and short-term plasticity by Munc13 isoforms. Neuron. 2002;33(3):411-424.

50. Yoon H, Enquist LW, Dulac C. Olfactory inputs to hypothalamic neurons controlling reproduction and fertility. Cell. 2005;123(4):669-682.

51. Lu J, Sherman D, Devor M, Saper CB. A putative flip-flop switch for control of REM sleep. Nature. 2006;441(7093):589-594.

52. Schoppa NE, Westbrook GL. Glomerulus-specific synchronization of mitral cells in the olfactory bulb. Neuron. 2001;31(4):639-651.

53. Hanks TD, Ditterich J, Shadlen MN. Microstimulation of macaque area LIP affects decision-making in a motion discrimination task. Nat Neurosci. 2006;9(5):682-689.

54. Kanold PO, Kara P, Reid RC, Shatz CJ. Role of subplate neurons in functional maturation of visual cortical columns. Science. 2003;301(5632):521-525

55. Hadjipavlou G, Dunckley P, Behrens TE, Tracey I. Determining anatomical connectivities between cortical and brainstem pain processing regions in humans: a diffusion tensor imaging study in healthy controls. Pain. 2006;123(1-2):169-178. 


\section{Publish your work in this journal}

The Journal of Pain Research is an international, peer reviewed, open access, online journal that welcomes laboratory and clinical findings in the fields of pain research and the prevention and management of pain. Original research, reviews, symposium reports, hypothesis formation and commentaries are all considered for publication.

Submit your manuscript here: https://www.dovepress.com/journal-of-pain-research-journal

The manuscript management system is completely online and includes a very quick and fair peer-review system, which is all easy to use. Visit http://www.dovepress.com/testimonials.php to read real quotes from published authors. 